\title{
Efficacy of Nursing Role in Implementing New Protocol for Management Iron Deficiency Anemia in Pregnancy
}

\author{
Warda Helmy wassef, Atef Mohamed Darwish \& Howieda Ahmed Fouly. \\ Specialist's Nurse at Mabarra Hospital Assiut, Egypt. \\ Professor of Obstetrics \& Gynecology Faculty of Medicine, Assiut University, Egypt. \\ Lecturer of Obstetric \& Gynecology of Nursing Faculty of Nursing Assiut University, Egypt.
}

\begin{abstract}
Aim: To test the efficacy of nursing role in implementing new protocol for management iron deficiency anemia in pregnancy. Design: A prospective interventional longitudinal study. Setting: Conducted at Women's Health Assiut University Hospital. Sample size: 60 cases divided into two groups: (A) received iron supplementation with health education and (B) received iron supplementation without health education. Results: Reflects positive outcomes of oral lactoferrin fizzing with a statistically significant difference when compared with other two different routes (Total infusion dose and oral tablets) of iron supplementations. Conclusion: Oral iron supplementation plus proper health education during implementing of the new protocol for management iron deficiency anemia in pregnancy, confirmed that oral iron supplementations in this study has a similar effect as parenteral rout in improvement the hemoglobin, $\mathrm{MCV}$ and $\mathrm{MCH}$ which reflect the importance of health education introduced by nurse. Recommendation: Increase awareness of pregnant women about importance of iron supplementation accompanied with good sources of nutrition and encourage health facilities to detect IDA with pregnant women earlier during ANC visits.
\end{abstract}

\section{Keywords: Lactoferrin, Total Dose Infusion, Iron Deficiency Anemia, Pregnancy \& Role of nursing.}

\section{Introduction}

Iron deficiency occurred when the body loss iron or body requirements overdo absorption, and is often co-factorial causes. The common cases occur during childhood period due to rapid growth and erythroid development or during especial condition as premature or low birth weight infants, in addition to different stages of developments e.g. toddlers and preschool age, and during teenage (Bauer, 2014).

In Egypt, iron deficiency anemia (IDA) is because of poor consumption practices which considers mainly for the development of iron deficiency anemia that is a sign of poor health condition (Pasricha, 2013). During pregnancy, anemia is one of the most common medical complaints disturbing pregnancy especially and contributes considerably to morbidities and mortalities. It is responsible about sixty percentage of maternal mortality in poor developing countries and causes direct and indirect deaths from cardiac failure, hemorrhage, infection and preeclampsia (Auerbach, 2011).

Consequently, there are different arrangement of interventions which aimed to prevent and correct iron deficiency anemia. These include improve nutritive components. Such as support food with iron. The proper use of iron supplements will be a vital part of anemia control programs in most of references, but supplements had better to be viewed as one of numerous tools in the combat alongside iron deficiency anemia (Khalafallah, 2010).
In addition, role of nursing to health education for families with anemic disorders is often a hard and inspiring task. Mealtime planning must be based on the family's economical status, cultural outline, and food favorite's choices. Habitually this needs more than a momentary argument with the mother or usual caregiver about foods high in iron (Batten, 2015).

\section{Significance of the study}

The proper health education of pregnant women with iron deficiency anemia would fasten the clinical and laboratory improvement particularly if it provided by specialized nurse.

\section{Hypothesis}

H1: If the new protocol of management for "iron deficiency anemia" accompanied with health education, the level of anemia will be decreased.

H0: If the new protocol of management for "iron deficiency anemia" accompanied with health education, the level of anemia won't be affected.

\footnotetext{
Aim of the study

To assess the efficacy of nursing role in implementing new protocol for management iron deficiency anemia in pregnancy.

Methodology

Design

This study was a prospective interventional longitudinal study and the duration of study for every pregnant is $8 \mathrm{Wks}$.
} 


\section{Setting}

This study conducted at Women's Health Asyut University Hospital, Asyut, Egypt. At the outpatient clinic of antenatal care unit (for data collection, counseling and follow up for patient) and inpatient for total dose infusion and any emergent management.

\section{Subjects}

The subjects of this study consisted of 60 cases. All the cases of pregnant women are 4 month's to 8 months of pregnancy. The cases divided into 30 of pregnant women's were given iron supplementation without health education and another 30 were given iron supplementation and health education for comparison between them.

Eligible criteria for inclusion include: 1.Pregnant Women aged $>18$ years. 2. Hemoglobin $<10 \mathrm{~g} / \mathrm{dl} 3$ Gestational age between 14-28 weeks ₹- Singleton pregnancy ${ }^{\circ}$. Willingness to participate and signing the informed consent form. While excluded the following: 1. Anemia predominantly caused by factors other than IDA (e.g. anemia with untreated B12 or foliate deficiency, hemolytic anemia), 2. Iron overload or disturbances in utilization of iron (e.g. haemochromatosis and haemosidrosis). 3. Decompensated liver cirrhosis and active hepatitis (ALAT>3 times upper limit of normal). 4. Active acute or chronic infections (assessed by clinical judgment supplied with white Blood Cells (WBC) and $\mathrm{C}$ - reactive protein (CRP). 5. Rheumatoid arthritis with symptoms or signs of active

\section{Sample size}

- The sample size calculated based on absolute change in $\mathrm{Hb}$ from baseline to week 8 .

- There were 60 cases; the size of sample would be defined as the For comparing two means calculated based on absolute change in $\mathrm{Hb}$ from baseline to week 8 .

- There were 60 cases; the size of sample would be defined as Confidence interval (2sided) 95\% with Power $80 \%$,

- The ratio of G1: G2 11: 10.2 wit Mean $\pm \mathrm{SD}=(1.1$ \pm 1.2 ) with variance 1.21 and 1.44 for G1and G2 respectively

\section{Pilot study}

The pilot study done on $10 \%$ of total sample to test feasibility and reliability of the questionnaire. No modification was done on the questionnaire, therefore the cases of pilot study included in the study.

\section{Ethical considerations}

Research proposal was approved from ethical committee in the faculty of nursing. There was no risk for study subject during application of the research. The study will follow common ethical principle in clinical research. Written consent was obtained from patients who would be participated in the study, after explaining the nature and purpose the study and confidentiality was assured. Also, participants had the right to refuse to participate and or withdraw from the study without any rational any time. Study subject privacy was considered during collection of data.

\section{Tools of the study}

The Questionnaire was include the following

- Full personal history taking as shown below: Name, age, married status, special habits, phone number.

- Menstrual history including: 1st day of last menstrual period. Obstetric history including: number of normal deliveries, any complication associated to delivery.

- General examination including: general condition, weight gain, pallor, vital signs, pulse, blood pressure, temp, abdominal examination, were done by physician, laboratory investigation, complete blood picture, serum iron, serum ferritin, total iron binding capacity, and transferring saturation were done in the laboratory.

- Health education booklet : including nutrition supplementation rich in iron, methods of taking oral supplementation.....etc

\section{Intervention}

\section{Screening of anemic cases}

- At the first visit, the investigator, introduce herself to the patient. Next, explain the importance and aims of the study. Give information about iron deficiency anemia (IDA) in pregnant women, incidence of, complications for mothers and babies, and treatment of IDA.

- Then, ask patient to make complete blood picture, to discover the level of iron deficiency anemia if it present.

\section{Grouping of the participants}

The investigator will divide the participants into two groups e.g. group (A) and group (B) according to Hemoglobin level and receiving of oral supplementation for IDA accompanied with health education in case of group (A) and without health education in case of group (B). Then the investigator asked patient to sign the document of her investigations result to receive iron supplementation.

- After that, the investigator observe hemoglobin's Level, when it less than $>11 \mathrm{~g} / \mathrm{dl}$, the patient will be group (A) who will receive oral iron supplementation and health education.

- Group (B) who will receive oral iron supplementation without health education.

- The interview with the patient was taken about 30 minutes. Also, in the first visit, each pregnant woman, assessment of basic information and clinical evaluation was performed, including age, complete history of previous pregnancies, medical 
and surgical history. Detailed general, physical, and clinical examination was carried out (including maternal age, body weight, height, estimation of fetal age both through examination and solography and biochemical assay).

- The iron supplementation" the management of this study in the form of Food supplemental for pregnant (lactoferrin) $3.5 \mathrm{gm}$ twice /day and it's based on fast dissolving through direct swallowing per oral route with fruits flavor taste.

\section{Follow-up}

- Each woman with IDA had a follow-up visit after first 4 weeks then after 8 weeks, when all physical and clinical examinations and biochemical measurements were repeated. The investigator was teach patient how to use, dose, frequent, route, and duration, follow-up with telephone, after one month's asked the women to go the hospital and make complete blood picture, level of iron.

- For patients who couldn't be able to go the hospital the investigator, go to the house to take the blood sample and make complete blood picture, to determine the level of iron. Record the results in the document, and compare between results before and after the iron medication (Aggett, 2012).

- Later follow up was done as assessment for all women after one month the investigator, asked them about general condition their babies, type of labor, any complications for mother and baby. If any complications was present the investigator registered the observation in a document.

\section{Statistical analysis}

The data were tested for normality using the Anderson-Darling test and for homogeneity variances prior to further statistical analysis. Categorical variables were described by number and percent $(\mathrm{N}$, $\%$ ), where continuous variables described by mean and standard deviation (Mean, SD). Chi-square and fisher exact tests used to compare between categorical variables where compare between continuous variables by unpaired t-test. A two-tailed $\mathrm{p}<0.05$ was considered statistically significant. All analyses were performed with the IBM SPSS 20.0 software. 


\section{Results}

Table (1): Comparison between studied groups with Health Education and without Health Education (HE) as regards Demographic Data.

\begin{tabular}{|c|c|c|c|c|c|}
\hline & \multicolumn{2}{|c|}{ With H E } & \multicolumn{2}{|c|}{ Without H E } & \multirow{2}{*}{ P. value } \\
\hline & No & $\%$ & No & $\%$ & \\
\hline \multicolumn{6}{|l|}{ Mother age } \\
\hline Mean \pm SD & \multicolumn{2}{|l|}{$32.23 \pm 5.37$} & \multicolumn{2}{|l|}{$26.7 \pm 6.09$} & $0.000 * *$ \\
\hline \multicolumn{6}{|l|}{ Educational level } \\
\hline Illiterate & 4 & 13.3 & 7 & 23.3 & \multirow[t]{5}{*}{0.142} \\
\hline Read and write & 5 & 16.7 & 0 & 0.0 & \\
\hline Preparatory & 6 & 20.0 & 4 & 13.3 & \\
\hline Secondary & 10 & 33.3 & 14 & 46.7 & \\
\hline University & 5 & 16.7 & 5 & 16.7 & \\
\hline \multicolumn{6}{|l|}{ Mothers occupation } \\
\hline House wife & 18 & 60.0 & 15 & 50.0 & \multirow{2}{*}{0.436} \\
\hline Employed & 12 & 40.0 & 15 & 50.0 & \\
\hline \multicolumn{6}{|l|}{ Residence } \\
\hline Rural & 10 & 33.3 & 24 & 80.0 & \multirow{2}{*}{$0.000 * *$} \\
\hline Urban & 20 & 66.7 & 6 & 20.0 & \\
\hline Age of menarche & \multicolumn{2}{|c|}{$12.67 \pm 1.71$} & \multicolumn{2}{|c|}{$13.33 \pm 1.03$} & 0.072 \\
\hline Duration & \multicolumn{2}{|c|}{$5.3 \pm 1.12$} & \multicolumn{2}{|c|}{$4.8 \pm 1.16$} & 0.094 \\
\hline Interval & \multicolumn{2}{|c|}{$25.43 \pm 3.4$} & \multicolumn{2}{|c|}{$24.8 \pm 3.66$} & 0.490 \\
\hline \multicolumn{6}{|l|}{ Rhythm } \\
\hline Regular & 24 & 80.0 & 27 & 90.0 & \multirow[t]{2}{*}{0.278} \\
\hline Irregular & 6 & 20.0 & 3 & 10.0 & \\
\hline Gravidity & \multicolumn{2}{|c|}{$3.17 \pm 1.39$} & \multicolumn{2}{|c|}{$2.87 \pm 1.25$} & 0.378 \\
\hline Parity & \multicolumn{2}{|c|}{$2.1 \pm 1.23$} & \multicolumn{2}{|c|}{$1.7 \pm 1.15$} & 0.199 \\
\hline Abortion & \multicolumn{2}{|c|}{$0.07 \pm 0.26$} & & & 0.322 \\
\hline Still birth & & & & & 0.959 \\
\hline Spacing & & & & & $0.044 *$ \\
\hline No. of living children & & & & & 0.189 \\
\hline Pregnancy normal & & & & & \\
\hline YES & 27 & 90.0 & 27 & 90.0 & 1000 \\
\hline $\mathrm{NO}$ & 3 & 10.0 & 3 & 10.0 & \\
\hline Complication & & & & & \\
\hline YES & 1 & 3.3 & 0 & 0.0 & 0313 \\
\hline $\mathrm{NO}$ & 29 & 96.7 & 30 & 100.0 & \\
\hline if yes & & & & & \\
\hline Hypertension Anemia & 1 & 3.3 & 0 & 0.0 & - \\
\hline Gestational Age & & & & & 0.024 \\
\hline Normal Labor & & & & & \\
\hline YES & 11 & 36.7 & 12 & 40.0 & 0.791 \\
\hline $\mathrm{NO}$ & 19 & 63.3 & 18 & 60.0 & \\
\hline Complication & & & & & \\
\hline YES & 0 & 0.0 & 1 & 3.3 & 0.313 \\
\hline $\mathrm{NO}$ & 30 & 100.0 & 29 & 96.7 & \\
\hline
\end{tabular}

*Statistically significant difference $(p<0.05) * *$ Statistically significant difference $(p<0.01)$ 
Table (2): Comparison between studied group regarding complete blood picture (pre and post) health education.

\begin{tabular}{|l|c|c|c|c|c|c|}
\hline & \multicolumn{2}{|c|}{ Without HE } & & & & \\
\cline { 2 - 7 } & pre & post & P. value & $\begin{array}{c}\text { Pre- } \\
\text { medication }\end{array}$ & $\begin{array}{c}\text { Post- } \\
\text { medication }\end{array}$ & $\begin{array}{c}\text { P. } \\
\text { value }\end{array}$ \\
\hline Hemoglobin & $9.68 \pm 1.2$ & $10.52 \pm 1.04$ & $0.006^{* *}$ & $9.41 \pm 0.8$ & $10.64 \pm 0.85$ & $0.000^{* *}$ \\
\hline R.B.CS & $4.06 \pm 0.73$ & $4.46 \pm 0.76$ & $0.040^{*}$ & $4.08 \pm 0.55$ & $4.73 \pm 0.5$ & $0.000^{* *}$ \\
\hline Hematocrit & $34.92 \pm 6.71$ & $37.36 \pm 7.52$ & 0.193 & $41.78 \pm 3.83$ & $44.57 \pm 4.42$ & $0.012^{*}$ \\
\hline Platelets & $241.64 \pm 91.91$ & $240.88 \pm 93.92$ & 0.975 & $267.19 \pm 91.66$ & $295.98 \pm 92.79$ & 0.231 \\
\hline W.B.CS & $6.62 \pm 2.49$ & $7.52 \pm 2.35$ & 0.153 & $7.43 \pm 2.44$ & $8.35 \pm 2.22$ & 0.131 \\
\hline R.D.W & $13.22 \pm 1.38$ & $14.3 \pm 1.82$ & $0.021^{*}$ & $12.49 \pm 0.94$ & $13.34 \pm 0.67$ & $0.000^{* *}$ \\
\hline M.C.V & $73.52 \pm 12.09$ & $78.12 \pm 7.67$ & 0.084 & $73.03 \pm 5.28$ & $75.98 \pm 4.35$ & $0.022^{*}$ \\
\hline M.C.H & $25.76 \pm 2.74$ & $27.81 \pm 2.45$ & $0.003^{* *}$ & $25.91 \pm 3.68$ & $28.11 \pm 2.77$ & $0.011^{*}$ \\
\hline M.C.H.C & $31.1 \pm 3.44$ & $33.41 \pm 3.19$ & $0.009^{*}$ & $30.22 \pm 4.11$ & $32.62 \pm 3.2$ & $0.015^{*}$ \\
\hline
\end{tabular}

$*$ Statistically significant difference $(p<0.05) \quad * *$ Statistically significant difference $(p<0.01)$

Table (3): Comparison between studied groups regarding type (TDI Vs. Oral fizzing) of IDA medications.

\begin{tabular}{|l|c|c|c|c|c|c|}
\hline \multirow{2}{*}{$\mathbf{t}$} & TDI $^{\boldsymbol{\epsilon}}$ & Oral $^{\infty}$ (fizzing) & P. value & TDI & Oral $^{\infty}$ (fizzing) $^{\text {P. value }}$ \\
\cline { 2 - 8 } & Pre treatment & Pre treatment & & $\begin{array}{c}\text { 4 weeks after } \\
\text { treatment }\end{array}$ & $\begin{array}{c}\text { 4 weeks after } \\
\text { treatment }\end{array}$ & \\
\hline $\mathrm{HB}$ & $8.22+0.87$ & $9.68 \pm 1.2$ & $<0.001^{* *}$ & $10.29+0.86$ & $10.52 \pm 1.04$ & 0.189 \\
\hline $\mathrm{MCV}$ & $68.62+4.89$ & $73.52 \pm 12.09$ & $<0.001 * *$ & $82.72+4.86$ & $78.12 \pm 7.67$ & $<0.001^{* *}$ \\
\hline $\mathrm{MCH}$ & $21.69+3.97$ & $25.76 \pm 2.74$ & $<0.001^{* *}$ & $27+1.14$ & $27.81 \pm 2.45$ & $0.021^{*}$ \\
\hline
\end{tabular}

t-test used for mean comparisons

* Statistically significant difference $(p<0.05)$

** Statistically significant difference $(p<0.01){ }^{\epsilon}$ Total amount of cosmofer administered as a total dose infusion

${ }^{\infty}$ Oral fizzing sachet

Table (4): Comparison between studied groups regarding type (TDI Vs. Oral fizzing) of IDA medications.

\begin{tabular}{|c|c|c|c|c|c|c|}
\hline $\mathbf{t}$ & Oral(tablet $)^{\alpha}$ & Oral (fizzing) & P. value & $\begin{array}{c}\text { Oral } \\
{\text { (tablet })^{\alpha}}^{\alpha}\end{array}$ & $\begin{array}{c}\text { Oral } \\
\text { (fizzing) }\end{array}$ & P. value \\
\hline & Pre treatment & Pre treatment & & $\begin{array}{c}4 \text { weeks after } \\
\text { treatment }\end{array}$ & $\begin{array}{c}4 \text { weeks after } \\
\text { treatment }\end{array}$ & \\
\hline $\mathrm{HB}$ & $8.58+0.73$ & $9.68 \pm 1.2$ & $<0.001 * *$ & $9.51+0.77$ & $10.52 \pm 1.04$ & $<0.001 * *$ \\
\hline $\mathrm{MCV}$ & $70.28+3.92$ & $73.52 \pm 12.09$ & $0.050^{*}$ & $80.08+3.14$ & $78.12 \pm 7.67$ & 0.069 \\
\hline $\mathrm{MCH}$ & $22.58+1.72$ & $25.76 \pm 2.74$ & $<0.001 * *$ & $26.32+1.28$ & $27.81 \pm 2.45$ & $<0.001 * *$ \\
\hline
\end{tabular}

Table (1): Shows comparison between studied groups who received Health education and who don't received Health education. Mean age of for women with health education and without health education revealed a statistically significant difference at $\mathrm{P}$ value $=0.000$. For residence $80 \%$ of rural women received health education versus $20 \%$ of don't received HE from urban with a statistically significant difference at $\mathrm{P}$ value $=0.000$

Table (2): Shows comparison between (pre and post) results of complete blood picture investigation with health education vs health education. There is a statistically significant difference of Hemoglobin,
R.B.Cs, Hematocrit, R.D.W, M.C.V, M.C.H and M.C.H.C at $(\mathrm{P}$. value $=(0.000,0.000,0.012,0.000$, $0.022,0.011,0.015)$ respectively. The outcomes of complete blood picture with group who didn't receive health education. The investigations revealed a statistically significant difference with $\mathrm{Hb}, \mathrm{RBCs}$, R.D.W, M.C.H and M.C.H.C levels before and after treatment at $(\mathrm{P}$. value $=0.006,0.040,0.021,0.003 \&$ $0.009)$ respectively.

Table (3): Shows the comparison of pre and post 4 weeks blood picture outcomes after using of two different IDA medications (Total dose infusion (TDI) and Oral fizzing). HB Mean + SD after 4 weeks of 
TDI Vs. Oral fizzing was 10.29+0.86 Vs. 10.52 \pm 1.04 with no statistical significant difference. While MCV and $\mathrm{MCH}$ revealed a statistical significant difference at $(\mathrm{P}=0.001,0.021)$ respectively.

Table (4): Shows the comparison of pre and post 4 weeks blood picture outcomes after using of two different IDA medications (oral tablet and Oral fizzing). HB Mean + SD after 4 weeks of TDI Vs. Oral fizzing was $9.51+0.77$ Vs. $10.52 \pm 1.04$ with a statistically significant difference at $(\mathrm{P}=0.001)$ $\mathrm{MCH}$ was $26.32 \pm 1.28$ Vs. $27.81 \pm 2.45$ revealed a statistically significant difference at $(\mathrm{P}=0.001)$.

\section{Discussion}

Our study focused on the importance of iron deficiency supplementation through its aims: To assess nursing role as a health educator in application of a new protocol of management for IDA and to test the efficacy of IDA supplementation on outcome of anemia level.

\section{Demographic Characteristics}

Regarding demographic characteristics, this study, findings related to participants' age was $29.5 \pm 6.3$ years old which is similar to other studies (Abu Salem, et al., 2015) in which 29.09 \pm 6.6 . Similarly, (Tariq et al., 2015) in which mean group was 28 years. While as study done in Menoufia, Egypt by (Rezk et al 2015) reported that participants' age was 25.56 \pm 5.76 .this difference may be related to subdivisions or categories in that study into three groups one of them received Lactoferrin similar to our study. This finding demonstrates the high prevalence of iron deficiency anemia in middle young aged pregnant women.

Regarding gestational age, this study findings revealed $(16 \pm 1.46$. $)$ weeks. This finding similar to (Rezk et al., 2015) who reported that Mean \& SD gestational age $(16.14 \pm 1.85)$. For residence more than fifty percentage $56.7 \%$ were from rural areas. This findings similar to a study reported by (Abu salem, et al., 2015) in which rural residences were $56.7 \%$. Education level revealed that illiterate and basic level were $43.3 \%$ while the secondary \& university education were 16.7 and secondary education was the most percentage $56.7 \%$. These findings match with (Abu salem, et al., 2015) in which $66 \%$ and $44.6 \%$ for the mentioned levels. For occupation more than fifty percent $55 \%$ were house wives which was very close to (Abu salem, et al., 2015) study which reported fifty percent. The similarity may be interpreted by that the two studies done in Egypt which means the similarities originates from the similar characteristics in the same country.

Baseline of laboratory investigations

In this study, iron deficiency anemia was diagnosed on basis of HB level, based on WHO criteria
(Hemoglobin $<11 \mathrm{~g} / \mathrm{dL}$ ). We observed that the mean hemoglobin, MCV, MCH, serum iron, The baseline $\mathrm{HB}$ at the time of inclusion in the study was less than $10 \mathrm{gm} / \mathrm{dl}$, it was $9.41 \mathrm{gm} / \mathrm{dl}$ for oral iron group which was similar to (Ayub et al., 2008 \& Abu salem, et al., 2015) who reported a baseline HB 9.58 and 9.49 $\mathrm{gm} / \mathrm{dl}$ for participants respectively. Similarly, (Erhabor, et al., 2013) who assessed the prevalence of IDA among pregnant women and non -pregnant women, and reported that HB level was less than 11 g/dl. While a study reported by (Rezk, et al., 2015) it was less than the previous mentioned studies and revealed $8.03 \mathrm{gm} / \mathrm{dl}$. However, the study done in Italy by revealed higher basic of $\mathrm{Hb} 11.5 \mathrm{gm} / \mathrm{dl}$. This difference due to higher level of health services and socioeconomic status for Italian women.

In our study, baseline of mean corpuscular volume $(\mathrm{MCV})$ and mean corpuscular hemoglobin $(\mathrm{MCH})$ in the two studied groups either with health education or without health education 73.03 and 25.91 respectively. Similarly, MCV in the study reported by (Abu salem, et al., 2015) which was 74.78 but this study not reported $\mathrm{MCH}$ in its laboratory findings. In the same line, the study reported by (Erhabor, et al., 2013) who reported that $\mathrm{MCV}, \mathrm{MCH}$ and $\mathrm{MCHC}$ observed among the pregnant subjects was significantly lower, 70.9, 25.4 and 31.9.

\section{Outcomes of iron supplementation}

After oral iron supplementation (Lactoferrin) received by pregnant women, the outcomes complete blood picture revealed an increase of blood components' levels with a statistically significant difference in Hemoglobin, R.B.Cs, M.C.V, M.C.H, Hematocrit, R.D.W, and M.C.H.C. The studies matches with our study reported by (Mehedintu, et al., 2015) who reported that an increasing in $\mathrm{Hb}$ level after one month of using oral $100 \mathrm{mg}$ bovine lactoferrin twice a day, before meals. (Similarly, Paesano, et al., 2014) who reported that $\mathrm{HB}$ and RBCs were significantly increased after receiving of bovine lactoferrin (bLf) oral administration therapy $100 \mathrm{mg}$ of bLf twice a day.

Comparison of TDI, Oral fizzing and Oral tablets of Lactoferrin treatment

The comparison between three different routes (TDI, Oral fizzing \& Oral tablets) of lactoferrin treatment after 4 weeks of administration, revealed the following: Comparing of TDI with oral (fizzing) revealed a statistically significant different in $\mathrm{Hb}$ level which is increasing more in its mean with TDI without any statistically significant difference. In addition $\mathrm{MCV}$ and $\mathrm{MCH}$ revealed a statistically significant different in $\mathrm{MCV} \& \mathrm{MCH}$ levels which were increased more with TDI.

Comparing of Oral (fizzing) with Oral (tablet) revealed a statistically significant different in $\mathrm{Hb}$ 
level which is increasing more in its mean with a statistically significant difference. While MCV revealed an increased level with oral tablet more than Oral (tablet) without statistically significant difference. $\mathrm{MCH}$ revealed a statistically significant different with $\mathrm{MCH}$ level increased more with oral tablet.

These findings matched with a study of (Manjunath \& Veena, 2013) who reported on $\mathrm{MCV}$ and $\mathrm{MCH}$ revealed a statistically significant difference before and /or after start of treatment. Others comparing iron sucrose to oral iron (Choudhuary et al., 2016) who reported a statistically significant increase in $\mathrm{MCV}$ and $\mathrm{MCH}$ in parenteral group which was similar to our study. Similarly, (Abdullah et al., 2014) who reported increase in $\mathrm{MCV}$ and $\mathrm{MCH}$ in both study groups after 4 weeks of treatment but without statistically significant difference between the two study groups.

\section{Health Education About Nutritional Iron \\ Resources}

The nurse role within the aspect of health promotion has shifted from a disease model to a health model (Piper, 2009). The most important role is figured in teaching and providing pregnant women with information needed based on different condition and intend to behavioral changes to maintain health during pregnancy mainly those related to nutritional problems. In this study, the investigator used a health education and information to teach pregnant women with IDA how they improve their health status through following a nutritional guide. This nutritional guidance was in the form of booklet in a simple illustrative tool "booklet" to guide mothers to rich sources of iron supplants.

The investigator divided participants into two groups one group received health education with iron supplementation in addition to health education and other group not received any health education and follow the routine advices during antenatal visits. The findings of our study revealed that the mean $\mathrm{Hb}$, RBCs, Hematocrit, R.D.W, M.C.V, M.C.H, and M.C.H.C levels increased of intervention group with a statistically significant different before and after Lactoferrin with health education. These findings interpreted by positive effect of health education during iron supplementation. Similarly, a study by (Al-Tell, et al., 2010) which showed an improvement in hemoglobin level, after receiving off $\mathrm{HE}$ and also women's perception about iron supplementation which reported in a study of the effect of Nutritional Interventions on Anemic Pregnant Women's Health Using Health Promotion Model.

The advantages of oral iron in treatment of iron deficiency anemia during pregnancy was significant increase in hemoglobin level, helps to rebuild iron stores, helping the symptoms of anemia to subside at a faster rate and reduces the risk of developing anemia in subsequent pregnancies, wide safety profile.

Oral treatment is safe alternative for the treatment of anemia, being able to reduce the need for blood transfusion and its concomitant side effect such as anaphylactic shock, febrile and hemolytic reaction.

\section{Conclusion}

This study conclude that Oral iron supplementation plus proper health education during implementing of the new protocol for management iron deficiency anemia in pregnancy, confirmed that oral iron supplementations in this study has a similar effect as parenteral rout in improvement the hemoglobin, $\mathrm{MCV}$ and $\mathrm{MCH}$ which reflect the importance of health education introduced by nurse "the study hypothesis".

Oral iron supplementations in this study confirm that could be used widely as an alternative to parenteral methods of iron supplementations due to the similar effect of improvement in $\mathrm{Hb}, \mathrm{MCV}$ and $\mathrm{MCH}$ which reflect the importance of oral iron supplementation. So the women with iron deficiency anemia can have a good options to use oral or parenteral with similar effect according to their condition.

\section{Recommendations}

- Increase awareness of pregnant women about importance of iron supplementation accompanied with good sources of nutrition

- Encourage health facilities to detect IDA with pregnant women earlier during ANC visits

- Further researches should be done to generalize the results of iron supplementation with new form as oral supplements.

\section{References}

1. Abdullah, A., Qadir, S., Haq, A., Wagay M., \& Masrat, S., (2014): International Journal of basic and applied medical scinces .4b (2) 78-83.

2. Abu Salem, M., Mahrous, O., El Shazly, H., Ibrahem, R., Al-oshari, S., (2015): Epidemiology of iron-deficiency anemia among pregnant women in Menoufia Governorate, Egypt and Taiz Governorate, Yemen: acomparative study. Menoufia Med J 29:1005-1011@ 2017 Faculty of Medicine, Menoufia University1110-2098DOI: $\quad$ 10.4103/11102098.202491 1005-1011.

3. Aggett, P., (2012): Iron in Erdman JW, Macdonald LA, Zeiel SH ,eds.present knowledge in Nutrition. 10th ed. Washington, DC: WileyBlack weel, 506-8. 
4. Al-Tell, M., El-Guindi F., Soliman N., \& ElNana, H., (2010): Effect of Nutritional Interventions on Anemic Pregnant Women's Health Using Health Promotion Model. Med. J. Cairo Univ., 78 (2) 109-118.

5. Auerbach M., Pappadakis J., Bahrain H., et al., (2011): Safety and efficacy of rapidly administered (one hour) one grams of low molecular weight iron dextran(INFeD) for the treatment of iron deficient anemia. 2nd ed. London: Hodder Arnold; 2010. p.139.

6. Ayub, R., Tariq, N., Adil, M., Iqbal, M., Junaid, A., Jaferry, T., (2008): Efficacy and Safety of Total Dose Infusion of Low Molecular Weight Iron Dextran in the Treatment of Iron Deficiency Anemia During Pregnancy. J Coll Physicians Surg Pak 18 (7), 424-427. 72008.

7. Batten S., Kenner, C., et al., (2015): Maternal, Neo Natal, and Women's Health Nursing.

8. Bauer, K., (2014): Hematologic changes during pregnancy. http://www.up to date com.home.

9. Chowdhury, S., Rahman, M., Moniruddin, A.B.M ${ }^{3}$., (2016): Anemia in Pregnancy. Medicine Today, 4 (26), 1 49- 56.

10. Erhabor, O., Isaac, I., Isah, A., Udomah, F., (2013): IRON DEFICIENCY ANAEMIA AMONG ANTENATAL WOMEN IN SOKOTO, NIGERIA. British Journal of Medical and Health Sciences, Vol. 1 (4) 47-57.

11. Khalafallah A., Dennis A., Bates J., Bates G., Robertson I., Smith L., Ball M., Seaton D.., Brain T., Rasko J., (2010): prospective randomized, controlled trial of intravenous versus oral iron for moderate iron deficiency anaemia of pregnancy. J Intern Med. Sep;268(3):286-95. doi: 10.1111/j.1365-2796.2010.02251.x. Epub 2010 May 19.

12. Manjunath, S., Veena, H., et al., (2013): International joumal of reoroduction, contraception,obstetric and gynaecology, 3, 35558.

13. Mehedintu, C., Ionescu, O., Ionescu, S., Cirstoiu M., Dumitrascu, M., Bratila, E., Dumitrescu, R., Oprescu, D., Tătaru, C., Vladareanu, S., (2015): Iron Deficiency and Iron-Deficiency Anaemia In Pregnant women corrected by oral bovine lactoferrin administration. FARMACIA, Vol. 63, 6, 922-926.

14. Pasricha, S., Darakesmith, H., Bloack, J., Hipgrave, D., Biggs B., (2013): control of iron deficiency anemia in low and middle- income countries, blood. 121:260.

15. Piper, S., (2009): Health Promotion for Nurses, Theory and Practice, paper back, Routledge ISBN, p 256.
16. Rezk, M., Kandi, M., Dawood, R., Shaheen, A., Allam, A., (2015): Oral lactoferrin versus ferrous sulphate and ferrous fumerate for the treatment of iron deficiency anemia during pregnancy. Journal of Advanced Nutrition and Human Metabolism 2, e740.doi: 10.14800/janhm.740.

17. Tariq, N., Ayub, R., Ullah Khan, W., Ijaz, S., Alam A., (2015): Parenteral Iron Therapy in the Treatment of Iron Deficiency Anemia During Pregnancy: A Randomized Controlled Trial. Coll Physicians Surg Pak 25 (3), 193-197. 